\title{
Running Non-Minimal Inflation with Stabilized Inflation Potential
}

\author{
Digesh Raut*i† \\ Department of Physics and Astronomy, University of Alabama, \\ Tuscaloosa, Alabama 35487, USA \\ E-mail: drautecrimson.ua.edu

\section{Nobuchika Okada} \\ Department of Physics and Astronomy, University of Alabama, \\ Tuscaloosa, Alabama 35487, USA \\ E-mail: okadan@ua. edu
}

\begin{abstract}
In the context of the Higgs model involving gauge and Yukawa interactions with the spontaneous gauge symmetry breaking, we consider $\lambda \phi^{4}$ inflation with non-minimal gravitational coupling, where the Higgs field is identified as inflaton. Since the inflaton quartic coupling is very small, once quantum corrections through the gauge and Yukawa interactions are taken into account, the inflaton effective potential most likely becomes unstable. In order to avoid this problem, we need to impose stability conditions on the effective inflaton potential, which lead to not only non-trivial relations amongst the particle mass spectrum of the model, but also correlations between the inflationary predictions and the mass spectrum. For concrete discussion, we investigate the minimal $B-L$ extension of the Standard Model with identification of the $B-L$ Higgs field as inflaton. The stability conditions for the inflaton effective potential fix the mass ratio amongst the $B-L$ gauge boson, the right-handed neutrinos and the inflaton. This mass ratio also correlates with the inflationary predictions. In other words, if the $B-L$ gauge boson and the right-handed neutrinos are discovered in future, their observed mass ratio provides constraints on the inflationary predictions.
\end{abstract}

The 11th International Workshop Dark Side of the Universe 2015

14-18 December 2015

Kyoto, Japan

\footnotetext{
${ }^{*}$ Speaker.

${ }^{\dagger}$ This work is based on work [1].
} 


\section{Introduction}

Current understanding of the cosmic origin and the evolution is tha our universe went through a period of rapid accelerated expansion at the beginning, known as inflation. Inflation $[2,3,4,5]$ solves many serious problems of the Standard Big-Bang Cosmology, such as the horizon, flatness and monopole problems. More importantly, the primordial density fluctuation generated during inflation can seed the formation of large scale structure we see today. In a simple inflationary scenario known as slow-roll inflation, inflation is driven by a single scalar field (inflaton) that slowly rolls down to its potential minimum. At the end of inflation, the inflaton decays to Standard Model (SM) particles to reheat the universe through a process known as reheating.

Recently the Planck 2015 results [6] have set an upper bound on the tensor-to-scalar ratio as $r \lesssim 0.11$ while the best fit value for the spectral index $\left(n_{s}\right)$ is $0.9655 \pm 0.0062$ at $68 \%$ CL. Hence, the simple chaotic inflationary scenario are disfavored because their predictions for $r$ are too large. Among many inflation models, $\lambda \phi^{4}$ inflation with non-minimal gravitational coupling $\left(\xi \phi^{2} \mathscr{R}\right.$, where $\phi$ is inflaton, $\mathscr{R}$ is the scalar curvature, and $\xi$ is a dimensionless coupling) is a very simple model, which can satisfy the constraints by the Planck 2015 with $\xi \gtrsim 0.001$ [7].

A compelling inflation scenario would be where the inflaton field plays another important role in particle physics. As an example of such a scenario, we consider the general Higgs inflation model in the presence of non-minimal gravitational coupling, where the scalar Higgs field play the crucial role to spontaneously break the gauge symmetry of the model. We identify the Higgs field as inflation, for a simple example, see [8]. As in the SM, the Higgs field in the general Higgs model has the gauge, Yukawa and quartic Higgs interactions. For complete analysis of inflation scenario in the Higgs model, we consider the effective inflaton/Higgs potential by taking quantum corrections into account. In fact, we see that quantum corrections most likely cause an instability of the effective inflaton potential. Note that unless the non-minimal coupling $(\xi)$ is very large, the quartic inflaton coupling is very small [7]. Hence, quantum corrections to the effective potential are dominated by the gauge and Yukawa interactions. We consider the renormalization group (RG) improved effective potential described as $V(\phi)=\frac{1}{4} \lambda(\phi) \phi^{4}$, where $\phi$ denotes inflaton, and $\lambda(\phi)$ is the running quartic coupling satisfying the (one-loop) RG equation of the form,

$$
16 \pi^{2} \frac{d \lambda}{d \ln \mu} \simeq C_{g} g^{4}-C_{Y} Y^{4}
$$

Here, $g$ and $Y$ are the gauge and Yukawa couplings respectively, and $C_{g}$ and $C_{Y}$ are positive coefficients whose actual values are calculable once the particle contents of the model are defined. Since the quartic coupling is very small, we have neglected terms proportional to $\lambda$ ( $\lambda^{2}$ term and the anomalous dimension term). The solution to the RG equation is controlled by $g$ and $Y$, which are much larger than $\lambda$ and independent of $\lambda$. Therefore, we expect that unless the beta function is extremely small, the running inflaton quartic coupling $\lambda$ is driven to be negative in the vicinity of the inflation initial value, in other words, the effective potential has true minimum (far) away from the vacuum set by the Higgs potential at the tree-level.

A simple way to avoid this problem is to require the beta function to vanish at the initial inflaton value (the stationary condition of $\lambda$ with respect to $\phi$ ), namely, $C_{g} g-C_{Y} Y=0$. This condition leads to a relation between $g$ and $Y$, equivalently, a mass relation between the gauge boson and 
fermion in the Higgs model. Since the Higgs quartic coupling at low energy is evaluated by solving the RG equation, in which the gauge and Yukawa couplings dominate, the resultant Higgs mass also has a relation to the gauge and fermion masses. The stability of the effective potential also requires the positivity of the second derivative of the potential, which leads to another constraint on the gauge and Yukawa couplings. In the slow-roll inflation, the inflationary predictions are determined by the slow-roll parameters defined with the potential and its derivatives, and therefore, the inflationary predictions have a correlation with the mass spectrum of the Higgs model.

In order to explicitly show the mass relation and the correlation between the particle mass spectrum and inflationary predictions, we take the minimal $B-L$ model as an example. This model is a very simple, well-motivated extension of the SM, where the global $B-L$ (baryon number minus lepton number) in the SM is gauged. Three right-handed neutrinos and the $B-L$ Higgs field (which is identified as inflaton) are introduced for the cancellation of the gauge and gravitational anomaly and the $B-L$ gauge symmetry breaking, respectively. Associated with the $B-L$ gauge symmetry breaking, the $B-L$ gauge boson and the right-handed neutrinos acquire their masses. With the generation of the Majorana right-handed neutrino masses, the seesaw mechanism for the light neutrino mass generation is automatically implemented in this model. Analyzing the RG evolutions of the $B-L$ sector and the effective inflaton ( $B-L$ Higgs) potential, we show the particle mass spectrum and its correlation to the inflationary predictions. Through the correlation, the Planck 2015 results provide us with constraints on the particle mass spectrum.

\section{Non-minimal $\lambda \phi^{4}$ inflation at tree-level}

In the Jordan frame, the action of our inflation model is given by (hereafter we always work in the Planck unit, $M_{P}=M_{P l} / \sqrt{8 \pi}=1$, where $M_{P l}=1.22 \times 10^{19} \mathrm{GeV}$ is the Planck mass)

$$
\mathscr{S}_{J}=\int \mathrm{d}^{4} x \sqrt{-g}\left[-\frac{1}{2}\left(1+\xi \phi^{2}\right) \mathscr{R}+\frac{1}{2}(\nabla \phi)^{2}-\frac{1}{4} \lambda \phi^{4}\right]
$$

where $\xi$ being a positive, dimensionless parameter. Using the conformal transformation, $g_{E \mu \nu}=$ $\left(1+\xi \phi^{2}\right) g_{\mu v}$, the action in the Einstein frame with canonical gravity sector is described as

$$
\mathscr{S}_{E}=\int \mathrm{d}^{4} x \sqrt{-g_{E}}\left[-\frac{1}{2} \mathscr{R}_{E}+\frac{1}{2}\left(\nabla_{E} \sigma\right)^{2}-V_{E}(\sigma)\right],
$$

In Einstein frame with canonical kinetic term, $\sigma(\phi)$ is the new inflaton field such that $\left(\sigma^{\prime}\right)^{2}=$ $\left(1+\xi \phi^{2}(1+6 \xi)\right) /\left(1+\xi \phi^{2}\right)^{2}$, where a prime denotes the derivative with respect to original Jordan field $\phi$. Inflaton potential in terms of $\phi$ is given by

$$
V_{E}(\sigma(\phi))=\frac{1}{4} \frac{\lambda \phi^{4}}{\left(1+\xi \phi^{2}\right)^{2}}
$$

The inflationary slow-roll parameters in terms of $\phi$ are expressed as

$$
\varepsilon(\phi)=\frac{1}{2}\left(\frac{V_{E}^{\prime}}{V_{E} \sigma^{\prime}}\right)^{2}, \quad \eta(\phi)=\frac{V_{E}^{\prime \prime}}{V_{E}\left(\sigma^{\prime}\right)^{2}}-\frac{V_{E}^{\prime} \sigma^{\prime \prime}}{V_{E}\left(\sigma^{\prime}\right)^{3}} .
$$


Amplitude of the curvature perturbation $\Delta_{\mathscr{R}^{2}}$ and the number of e-folds $N$ are given by

$$
\Delta_{\mathscr{R}}^{2}=\left.\frac{V_{E}}{24 \pi^{2} \varepsilon}\right|_{k_{0}}, N=\frac{1}{2} \int_{\phi_{\mathrm{e}}}^{\phi_{I}} \frac{d \phi}{\sqrt{\varepsilon(\phi)}}\left(\frac{d \sigma}{d \phi}\right)
$$

where $\Delta_{\mathscr{R}}^{2}=2.195 \times 10^{-9}$ from the Planck 2015 results [6] with the pivot scale chosen at $k_{0}=0.002$ $\mathrm{Mpc}^{-1}, \phi_{I}$ is the inflaton value at horizon exit corresponding to the scale $k_{0}$, and $\phi_{e}$ is the inflaton value at the end of inflation, which is defined by $\max \left[\varepsilon\left(\phi_{e}\right),\left|\eta\left(\phi_{e}\right)\right|\right]=1$. The value of $N$ depends logarithmically on the energy scale during inflation as well as on the reheating temperature, and is typically around 50-60. The slow-roll approximation is valid as long as the conditions $\varepsilon \ll 1$ and $|\eta| \ll 1$ hold. In this case, the inflationary predictions, the scalar spectral index $n_{s}$ and the tensor-to-scalar ratio $r$ are given by

$$
n_{s}=\left.(1-6 \varepsilon+2 \eta)\right|_{\phi=\phi_{I}}, \quad r=\left.16 \varepsilon\right|_{\phi=\phi_{I}} .
$$

\section{Running $B-L$ Higgs inflation and stability of inflaton potential}

\begin{tabular}{c|ccc|c} 
& $\mathrm{SU}(3)_{c}$ & $\mathrm{SU}(2)_{L}$ & $\mathrm{U}(1)_{Y}$ & $\mathrm{U}(1)_{B-L}$ \\
\hline$N R^{i}$ & $\mathbf{1}$ & $\mathbf{1}$ & 0 & -1 \\
\hline$H$ & $\mathbf{1}$ & $\mathbf{2}$ & $-1 / 2$ & 0 \\
$\varphi$ & $\mathbf{1}$ & $\mathbf{1}$ & 0 & +2
\end{tabular}

Table 1: Particle contents of the $B-L$ sector.

We consider minimal extension B-L extension of SM with scalar field $\varphi$ and three generations of degenerate right-handed neutrinos $N R^{i}$, for simplicity. The $B-L$ gauge symmetry is broken by the vacuum expectation value (VEV) of $\varphi$ in its Higgs potential of $V(|\varphi|)=\lambda\left(\varphi^{\dagger} \varphi-\frac{v_{B L}}{2}\right)^{2}$, where $\varphi=\left(v_{B L}+\phi\right) / \sqrt{2}$ in the unitary gauge with the physical Higgs filed $\phi$ identified as inflaton. Associated with the gauge symmetry breaking, the right-handed neutrinos acquire their Majorana masses through the Yukawa interactions, $\mathscr{L} \supset-\frac{1}{2} \sum_{i=1}^{3} Y \varphi \overline{N R^{i c}} N R^{i}+$ h.c.. After the $B-L$ symmetry breaking with the Higgs $\operatorname{VEV}\langle\varphi\rangle=v_{B L} / \sqrt{2}$, the particle masses are given by

$$
m_{Z^{\prime}}=2 g v_{B L}, m_{N R}^{i}=\frac{1}{\sqrt{2}} Y v_{B L}, m_{\phi}=\sqrt{2 \lambda} v_{B L} .
$$

For inflationary consideration, with inflation scale close to Planck mass, we neglect $v_{B L}$ so the potential is given by $V(\phi)=\frac{1}{4} \lambda(\phi) \phi^{4}$. In the Einstein frame, the RG improved effective inflaton potential at the one-loop level is then given by $V_{E}(\phi)=\frac{1}{4} \lambda(\Phi) \Phi^{4}$, where $\Phi \equiv \phi / \sqrt{1+\xi \phi^{2}}$ [9]. The RG equations of the couplings at the one-loop level are given by ${ }^{1}[8]$

$$
\begin{aligned}
& 16 \pi^{2} \mu \frac{d g}{d \mu}=13 g^{3}, \\
& 16 \pi^{2} \mu \frac{d Y}{d \mu}=-6 g^{2} Y+\frac{5}{2} Y^{3}, \\
& 16 \pi^{2} \mu \frac{d \lambda}{d \mu}=20 \lambda^{2}-\left(48 g^{2}-6 Y^{2}\right) \lambda+96 g^{4}-3 Y^{4} .
\end{aligned}
$$

\footnotetext{
${ }^{1}$ There are a few different prescriptions for computing quantum corrections in the presence of the non-minimal gravitational coupling, for recent detailed computations of quantum corrections, see [9].
} 

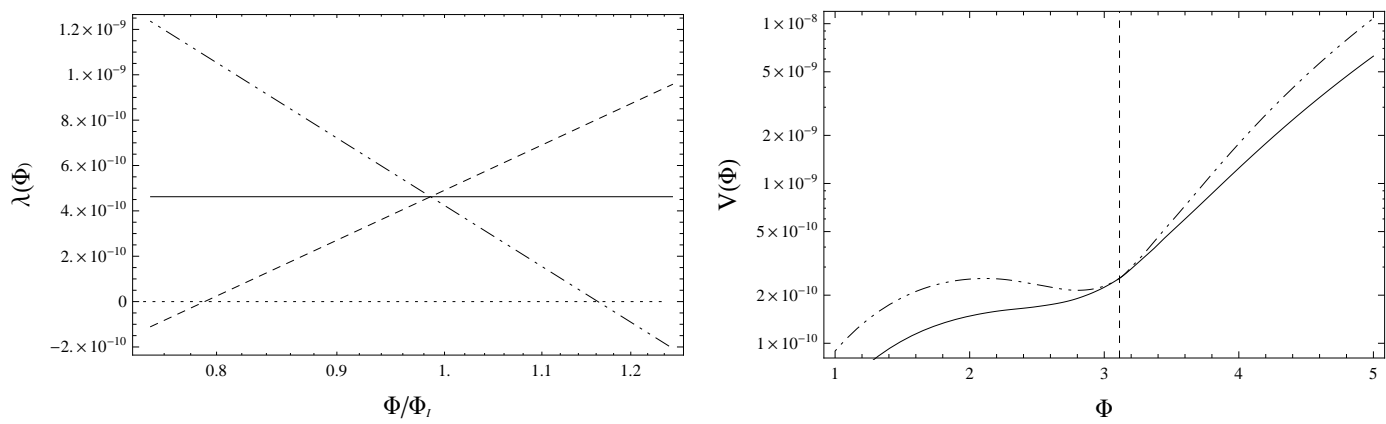

Figure 1: Left panel shows RG evolution of the quartic coupling $\lambda$ for fixed $Y=0.0237$ and $\xi=1$ and various values of $g=0.01$ (solid), 0.011 (dashed) and 0.009 (dot-dashed). The stability condition of $\beta_{\lambda}\left(\Phi_{I}\right)=0$ is satisfied for $g=0.01$ (solid line). Right panel shows RG improved effective potential for two values of $g$ and a fixed $\xi=0.1$, such that Yukawa is fixed by $\beta_{\lambda}\left(\Phi_{I}\right)=0$ condition. The solid and dot-dashed lines are the RGE improved potential for $g=0.041,0.046$ are respectively. For $g=0.046>g_{\max }=0.0425$, the local minimal is developed. The vertical dashed line indicates $\Phi=\Phi_{I}$ (in the Planck unit).

Let us now investigate the stability of the effective inflaton potential. In our analysis throughout this paper, we set the initial values of $\lambda$ to be the one obtained in the tree-level analysis at the initial inflaton value $\phi=\phi_{I}$, equivalently, $\Phi_{I}=\phi_{I} / \sqrt{1+\xi \phi_{I}^{2}}$. Then, we consider the RG improved effective inflaton potential by taking into account the RG evolution of the quartic coupling with the initial condition at $\phi_{I}$. The inflaton quartic coupling is very small unless $\xi \gg 1$ (see [1] for details). Hence the beta function of the quartic coupling is approximately given by

$$
\beta_{\lambda} \simeq \frac{1}{16 \pi^{2}}\left(96 g^{4}-3 Y^{4}\right)
$$

when $g^{2}, Y^{2} \gg \lambda^{2}$. The RG evolution is controlled by $g$ and $Y$, independent of the initial value of the inflaton quartic coupling. Fig. 1 shows the RG evolution of the quartic coupling in the vicinity of the initial inflaton value for various values of $g$ and $Y$ with a fixed $\xi=1$. We can see from Fig. 1 (left panel) that if the condition of $\beta_{\lambda}=0$ is violated even with $\pm 10 \%$ deviations for the values of $g$ for fixed Yukawa coupling value, the running quartic coupling quickly becomes negative in the vicinity of $\Phi_{I}$ (see the dashed and the dot-dashed lines). Same is true if gauge coupling is fixed and Yukawa is varied. This fact indicates that the $B-L$ gauge symmetry breaking vacuum at $\phi=v_{B L}$ is unstable and the effective potential develops a true vacuum with a negative cosmological constant.

In order to avoid this instability, we impose $\beta_{\lambda}=0$ and $d \beta_{\lambda} / d \Phi>0$ at $\Phi_{I}$. From the first condition, the Yukawa coupling $Y$ is determined by the gauge coupling, which we take as a free parameter in our analysis, along with the others, $\xi$ and $v_{B L}$. The second condition ensures that the effective potential is monotonically increasing in the vicinity of $\Phi_{I}$, and yields a lower bound on $g>g_{\min }$. When we analyze the global structure of the effective potential, we can notice that there exists an upper bound on $g<g_{\max }$. For a large $g>g_{\max }$, the effective potential develops a local

\footnotetext{
${ }^{2}$ In this paper, we are interested in this case, otherwise the beta function is so small that the inflaton quartic coupling is almost RG invariant. Although the tree-level analysis is valid in this case, the gauge and Yukawa couplings are too small to yield any impacts in the experimental point of view.
} 
minimum at $\Phi<\Phi_{I}$, so that the inflaton field will be trapped in this minimum after inflation. To avoid this problem, the parameter region is restricted to be in the range of $g_{\min }<g<g_{\max }$. Fig. 1 shows, for fixed $\xi=0.1$, effective potentials the gauge coupling $g=0.041,0.046$, such that first one is smaller while the second one is larger than $g_{\max }=0.0425$.

\section{Inflationary predictions and low energy observables}

Under the stability conditions, $g_{\min }<g<g_{\max }$ with various value of $\xi$, we now calculate the inflationary predictions with the effective inflaton potential. The quantum corrections through the gauge and Yukawa coupling completely change our inflationary scenario from the one at the tree-level. Since we refer the results in the tree-level analysis for $\lambda\left(\Phi_{I}\right)$ for a fixed $\xi$ and impose the stability condition $\beta_{\lambda}\left(\Phi_{I}\right)=0$, our prediction for the tensor-to-scalar ratio $r$ is the same as the one obtained in the tree-level analysis. However, the RG evolution of the inflaton quartic coupling alters the other inflationary predictions, $n_{s}$, from those obtained in the tree-level analysis, because they are calculated by the second and third derivatives of the effective potential (see [1] for details).

In our model, with a fixed e-folding number $N=50 / 60$, there are only three free parameters, $\xi, v_{B L}$ and $g$. For a choice of $\xi$ and fixed $N=60, \Phi_{I}$ and $\lambda\left(\Phi_{I}\right)$ are determined by the tree-level analysis, so that the inflationary prediction, except for $r$, is controlled by the gauge coupling $g$. In the $B-L$ model, the particle mass spectrum is determined by the gauge, Yukawa and inflation quartic couplings at the scale $v_{B L}$ (see Eq. (3.1)), which are obtained by solving the RG equations in Eq. (3.2). Considering all of these facts, we can expect a nontrivial mass relation in the particle spectrum and a nontrivial correlation between the inflationary predictions and the particle mass spectrum. For simplicity, we fix $v_{B L}$ so as to yield $m_{Z^{\prime}}=2 g\left(v_{B L}\right) v_{B L}=3 \mathrm{TeV}$, to be consistent with the current results from the search for $Z^{\prime}$ boson resonance at the Large Hadron Collider [10, 11].

In our paper[?] we have analytically shown that for $\xi \gtrsim 1$, the deviation of the predicted $n_{s}$ value from the tree-level analysis becomes smaller, as shown in Fig. 2. Hence we are interested in cases $\xi \lesssim 1$ so that $g_{\max } \ll 1$, and so is the corresponding $Y$. Hence both $g$ and $Y$ are almost RG invariant and the mass ratio $m_{N} / m_{Z^{\prime}} \simeq 0.84$ is almost independent of $\xi$ and is determined by $Y\left(\Phi_{I}\right) / g\left(\Phi_{I}\right)$. With fixed $m_{Z^{\prime}}=3 \mathrm{TeV}, v_{B L} \propto 1 / g\left(\Phi_{I}\right)$ and the same applies to the inflaton mass $m_{\phi}$ as shown in Fig. 2 (left panel), with the input values of $g$ in the range of $g_{\min }<g<g_{\max }$.

Also, in Fig. 2 (right panel), we show inflationary predictions for various $\xi \lesssim 1$ values, along with the contours given by the Planck 2015 results, with $m_{Z^{\prime}}=3 \mathrm{TeV}$. As discussed above, the tensor-to-scalar ratio is invariant while the predicted spectral index is altered by quantum corrections. Also, we can see that for $\xi \lesssim 1$, the results show sizable deviations for $g \sim g_{\text {max }}$ from those at the tree-level analysis depicted as the diagonal dashed line. Interestingly, the Planck 2015 results provide upper bounds on $g$, which are more severe than $g_{\max }$ for $\xi \lesssim 0.001$. The contour lines (diagonal solid lines) are inflationary predictions for with various fixed $g\left(v_{B L}\right)$ values.

\section{Conclusions}

We have considered the general Higgs model with the gauge and Yukawa interactions with the spontaneous gauge symmetry breaking. In the presence of the non-minimal gravitational coupling, 

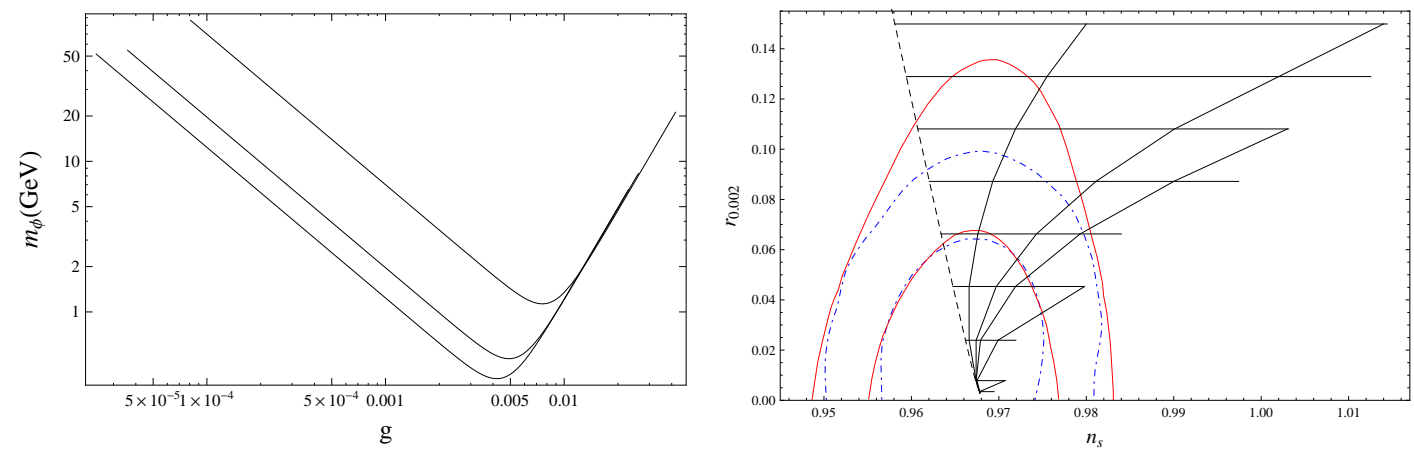

Figure 2: Low energy observables and inflationary predictions for various $\xi \lesssim 1$, with fixed $N=60$ and $m_{Z^{\prime}}=3 \mathrm{TeV}$, such that $g_{\min }<g<g_{\max }$. Left panel shows mass of inflaton $m_{\phi}$, with $\xi \simeq 0.0029,0.01$ and 0.1 respectively from top to bottom. Right panel shows the inflationary predictions $n_{s}$ and $r$ for various values of $\xi \lesssim 1$, along with the contours at the confidence levels of $68 \%$ and $95 \%$ given by the results of Planck 2015 (solid) and Planck+BICEP2/Keck Array (dot-dashed) [6]. The diagonal dashed line denotes the inflationary predictions in the tree-level analysis. The horizontal solid lines from top to bottom correspond to the results for $\xi=1.5 \times 10^{-3}, 2.1 \times 10^{-3}, 2.9 \times 10^{-3}, 4.1 \times 10^{-3}, 6 \times 10^{-3}, 0.01,0.02,0.1$ and 1. Diagonal solid lines are contours for fixed $g\left(v_{B L}\right)=0.0184,0.0216,0.023,0.026$, and 0.0425 ).

the Higgs field can also play the role of inflaton. The analysis with the Higgs potential at the treelevel leads to the inflationary predictions consistent with the cosmological observations. However, once we take quantum corrections, the effective inflaton potential most likely becomes unstable. This is because the inflaton quartic coupling is extremely small in a large portion of the parameters space and the effective potential is controlled by the gauge and Yukawa couplings independently of the quartic coupling. In the renormalization group improved effective potential, we see that the running quartic coupling becomes negative in the vicinity of the initial inflaton value, indicating the instability of the effective potential. In order to avoid this problem, we have imposed the stability condition of vanishing the beta function of the inflation quartic coupling. This condition leads to a non-trivial relation between the gauge and fermion masses. Since the renormalization group evolution of the inflaton quartic coupling is mainly controlled by the gauge and Yukawa coupling, the inflation mass at low energy is determined by the couplings. Therefore, the mass spectrum of the gauge boson, fermion and inflation shows a non-trivial relation.

Since the inflaton potential is modified from the tree-level one, the inflationary predictions are altered from those obtained by the tree-level analysis. Although the prediction of the tensor-toscalar ratio remains the same under the condition of the vanishing beta function, the predictions for the scalar spectral index and the running of the spectral index can be significantly altered. The fact that the effective potential is controlled by the gauge and Yukawa couplings implies a correlation between the inflationary predictions and the particle mass spectrum. Therefore, the observables at the gauge symmetry breaking scale correlate with the inflationary predictions which determined by physics at an extremely high energy compared to the gauge symmetry breaking scale.

By taking the minimal $B-L$ extension of the Standard Model as a simple example, we have shown such a non-trivial relation in the particle mass spectrum driven by the stability condition of the effective inflaton potential. We also have calculated the inflationary predictions from the 
effective potential and found their dependence of the $B-L$ gauge coupling. Therefore, the new particle mass spectrum of the $B-L$ model, once observed, has an implication to the inflationary predictions. On the other hand, more precise measurements of the inflationary predictions yield a constraint on the $B-L$ particle mass spectrum.

\section{Acknowledgements}

This work is supported in part by the United States Department of Energy Grant, No. DE-SC 0013680 .

\section{References}

[1] N. Okada, and D. Raut, Running Non-Minimal Inflation with Stabilized Inflaton Potential, [arXiv:1509.04439 [hep-ph]].

[2] A. A. Starobinsky, A New Type of Isotropic Cosmological Models Without Singularity, Phys. Lett. B 91 (1980) 99.

[3] A. H. Guth, The Inflationary Universe: A Possible Solution to the Horizon and Flatness Problems, Phys. Rev. D 23 (1981) 347.

[4] A. D. Linde, Chaotic Inflation, Phys. Lett. B 129 (1983) 177.

[5] A. Albrecht and P. J. Steinhardt, Cosmology for Grand Unified Theories with Radiatively Induced Symmetry Breaking, Phys. Rev. Lett. 48 (1982) 1220.

[6] P. A. R. Ade et al. [Planck Collaboration], Planck 2015 results. XIII. Cosmological parameters, [arXiv:1502.01589 [astro-ph.co]].

[7] N. Okada, M. U. Rehman and Q. Shafi, Tensor to Scalar Ratio in Non-Minimal $\phi^{4}$ Inflation, Phys. Rev. D 82 (2010) 043502, [arXiv: 1005.5161 [hep-ph] ]; N. Okada, V. N. Senoguz and Q. Shafi, The Observational Status of Simple Inflationary Models: An Update, [arXiv: 1403.6403 [hep-ph ] ]; T. Inagaki, R. Nakanishi and S. D. Odintsov, Non-Minimal Two-Loop Inflation, Phys. Lett. B 745 (2015), [arXiv:1502.06301 [hep-ph]].

[8] N. Okada, M. U. Rehman and Q. Shafi, Non-Minimal B-L Inflation with Observable Gravity Waves, Phys. Lett. B 701 (2011) 520, [arXiv:1102.4747 [hep-ph] ].

[9] D. P. George, S. Mooij and M. Postma, Quantum corrections in Higgs inflation: the real scalar case, JCAP 1402 (2014) 024, [arXiv:1310.2157 [hep-th] ].

[10] G. Aad et al. [ATLAS Collaboration], Search for high-mass dilepton resonances in pp collisions at $\sqrt{s}=8 \mathrm{TeV}$ with the ATLAS detector, Phys. Rev. D no.5 90 (2014) 052005, [arXiv: 1405.4123 [hep-ex]].

[11] CMS Collaboration [CMS Collaboration], Search for Resonances in the Dilepton Mass Distribution in pp Collisions at sqrt(s) = 8 TeV, CMS-PAS-EXO-12-061 (2013); V. Khachatryan et al. [CMS Collaboration], Search for physics beyond the standard model in dilepton mass spectra in proton-proton collisions at $\sqrt{s}=8 \mathrm{TeV}$, JHEP 1504 (2015) 025. 